Supporting Information:

\title{
Nonvolatile optically reconfigurable radiative metasurface with visible tunability for anti- counterfeiting
}

Ziquan Xu, ${ }^{1}$ Hao Luo, ${ }^{1}$ Huanzheng Zhu, ${ }^{1}$ Yu Hong, ${ }^{1}$ Weidong Shen, ${ }^{1}$ Jianping Ding, ${ }^{1}$ Sandeep Kaur, ${ }^{1}$ Pintu Ghosh, ${ }^{1}$ Min Qiu,,${ }^{2,3}{ }^{*}$ and Qiang $L i{ }^{1, *}$

${ }^{1}$ State Key Laboratory of Modern Optical Instrumentation, College of Optical Science and Engineering, Zhejiang University, Hangzhou 310027, China

${ }^{2}$ Key Laboratory of 3D Micro/Nano Fabrication and Characterization of Zhejiang Province, School of Engineering, Westlake University, 18 Shilongshan Road, Hangzhou 310024, China

${ }^{3}$ Institute of Advanced Technology, Westlake Institute for Advanced Study, 18 Shilongshan Road, Hangzhou 310024, China 


\section{S1 Supporting Information Methods}

\section{S1.1 Device fabrication}

$\mathrm{Au}$ was thermally evaporated onto a single-side polished and undoped silicon substrate. $\mathrm{ZnS}$ was then electrically evaporated. $\mathrm{A} \mathrm{Ge}_{2} \mathrm{Sb}_{2} \mathrm{Te}_{5}$ target was used in the sputtering of the GST film. An $\mathrm{Al}_{2} \mathrm{O}_{3}$ capping layer was electrically evaporated on top of the GST layer for protection. The deposition rates are $1.5 \mathrm{~nm} / \mathrm{s}(\mathrm{ZnS}), 0.52 \mathrm{~nm} / \mathrm{s}$ (GST), and $0.06 \mathrm{~nm} / \mathrm{s}\left(\mathrm{Al}_{2} \mathrm{O}_{3}\right)$, respectively.

\section{S1.2 Laser direct writing technique}

For the laser pulses control, a $450 \mathrm{~nm}$ laser diode was mounted on a TEC-cooled mount with an integrated bias-T adapter (Thorlabs, LDM56/M) to allow both DC and RF modulation of laser pulses. An arbitrary waveform generator (Agilent, 81150A) was externally triggered to generate the pumping pulses. The laser beam was tightly focused using a $100 \mathrm{X}$ objective lens $(\mathrm{NA}=0.9)$. For the position control, a 2 -axis stepping motor stage and a 3-axis piezo scanning stage are combined to obtain highprecision positioning and a large travel range.

\section{S1.3 Measurements}

For IR absorptance, the reflectance spectra are measured using an IR microscope (Bruker, Hyperion 1000, with the liquid-nitrogen-cooled MCT detector) connected to a FT-IR spectrometer (Bruker, Vertex 70). The MWIR images and LWIR images are taken by two IR cameras equipped with microscope lenses in spectral ranges of $3.0-$ $5.0 \mu \mathrm{m}$ (Telops, M200) and $7.7-9.3 \mu \mathrm{m}$ (Telops, L200), respectively. During the measurements, the sample is heated to $80{ }^{\circ} \mathrm{C}$ to increase the intensity of emitted IR 
light from the radiative metasurface which will also suppress the influence of background thermal emission.

For visible reflectance and scattering measurements, the sample is characterized by a spectrometer (Ocean Optics, QE65 Pro) connected to a microscope (Nikon, Eclipse 80i). In case of reflectance measurement, a protected Ag mirror is used as a reference. To measure the scattering, the microscope is switched at dark-field mode to block the specular reflection.

\section{S1.4 Calculation and simulation}

The radiative metasurfaces and the sub-micron-sized bumps and the corresponding spectra features are modeled and calculated in commercial FDTD software (Lumerical FDTD) with relative permittivity parameters of $\mathrm{Au}^{\mathrm{S} 1}, \mathrm{ZnS}^{\mathrm{S} 2}, \mathrm{GST}^{\mathrm{S} 3}$, and $\mathrm{Al}_{2} \mathrm{O}_{3}{ }^{\mathrm{S} 1}$ taken from literatures. See SI, Note 4 for details. 


\section{S2 Supporting Information Notes}

S2.1 Background thermal emission in the measurement of emissive patterns using IR cameras

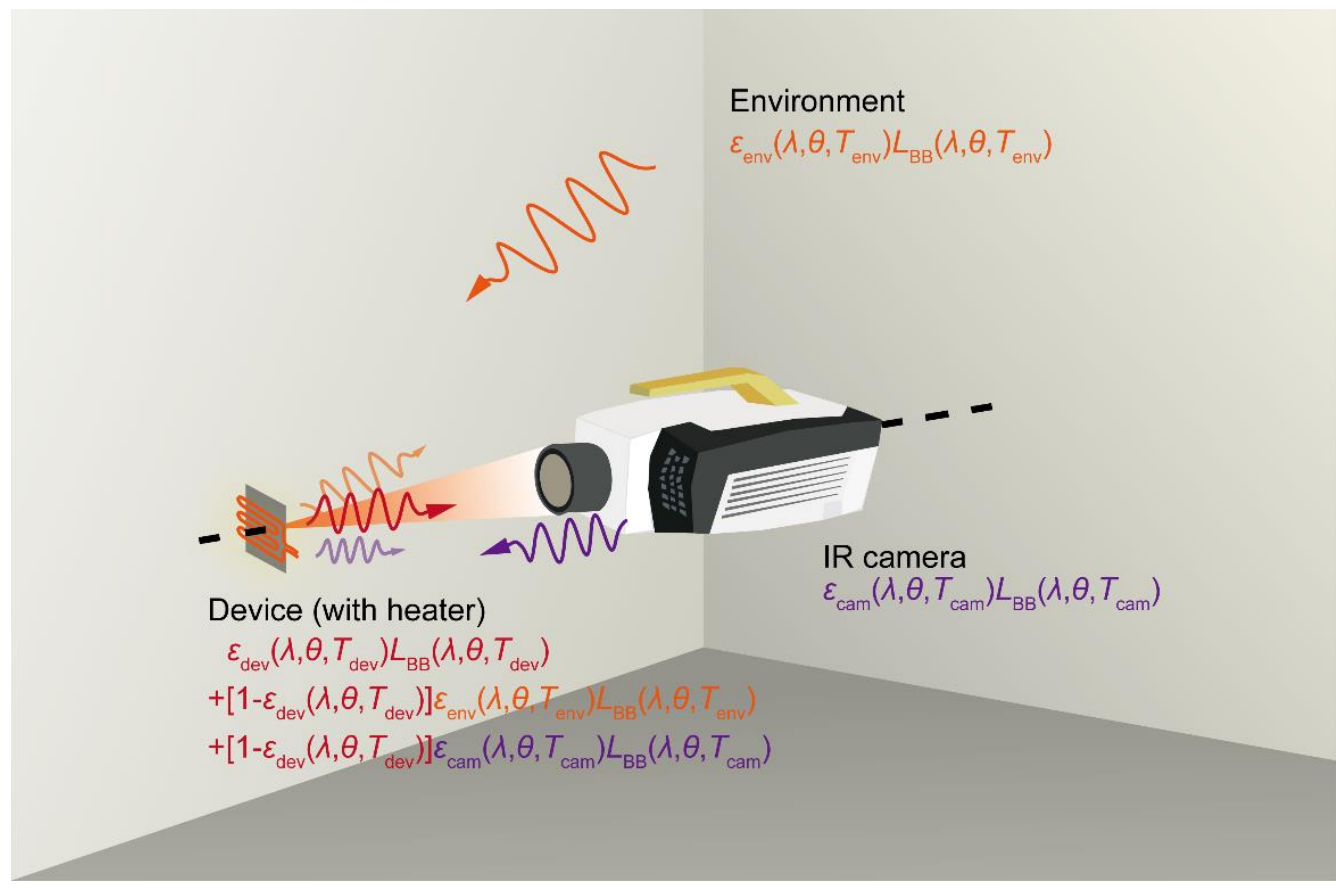

Figure S1. Thermal emission sources in the measurement of IR radiative metasurfaces.

Since the environment and the IR camera are also emissive in the IR spectral bands, it is of vital importance to count the background out when capturing the emissive patterns of the IR radiative metasurfaces. In the measurements, the radiative metasurface device, the IR camera and the environment are the three main sources of thermal emission (Figure S1). In this work, the characterization demands thermal emission from the IR radiative metasurfaces $L_{\mathrm{dev}}\left(\lambda, \theta, T_{\mathrm{dev}}\right)$, which can be expressed as

$$
L_{\mathrm{dev}}\left(\lambda, \theta, T_{\mathrm{dev}}\right)=\varepsilon_{\mathrm{dev}}\left(\lambda, \theta, T_{\mathrm{dev}}\right) L_{\mathrm{BB}}\left(\lambda, \theta, T_{\mathrm{dev}}\right)
$$


where $\varepsilon_{\mathrm{dev}}\left(\lambda, \theta, T_{\mathrm{dev}}\right)$ and $L_{\mathrm{BB}}\left(\lambda, \theta, T_{\mathrm{dev}}\right)$ are the spectral emissivity of the device and the radiance of the blackbody at the temperature of $T_{\mathrm{dev}}$, respectively.

The background thermal emission consists of two parts, the thermal emission from the environment $L_{\text {env }}\left(\lambda, \theta, T_{\text {env }}\right)$ and the thermal emission from the IR camera $L_{\text {cam }}\left(\lambda, \theta, T_{\text {cam }}\right)$. Specular reflections of the background thermal emission on the surface of the IR radiative metasurfaces are considered as the background thermal emission multiplies the reflectance of the device $R_{\mathrm{dev}}\left(\lambda, \theta, T_{\mathrm{dev}}\right)$, which equals to $1-A_{\mathrm{dev}}\left(\lambda, \theta, T_{\mathrm{dev}}\right)$ for non-transsmisive devices. According to the Kirchhoff's law of thermal radiation, the absorptance $A_{\mathrm{dev}}\left(\lambda, \theta, T_{\mathrm{dev}}\right)$ equals to the emissivity $\varepsilon_{\mathrm{dev}}\left(\lambda, \theta, T_{\mathrm{dev}}\right)$.

a

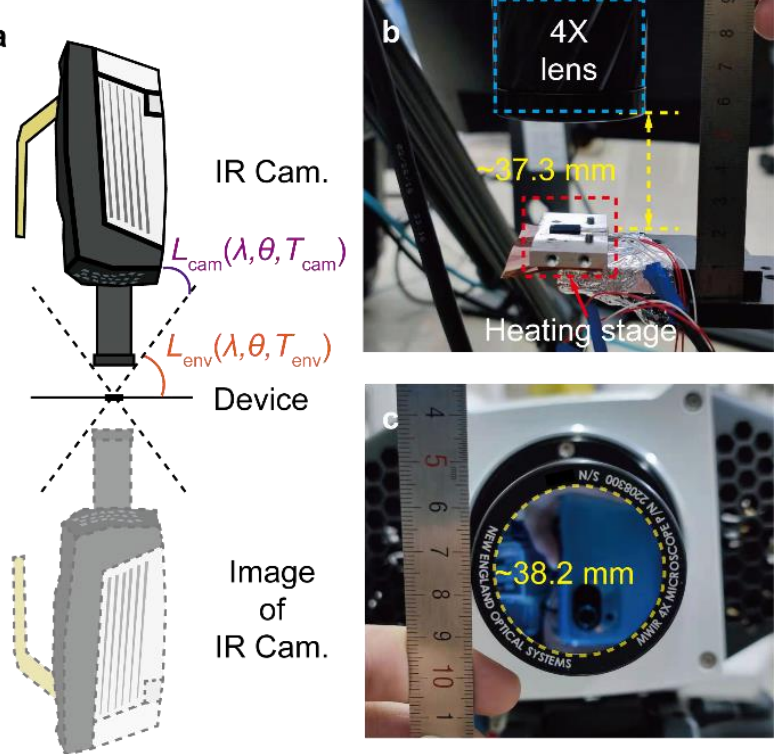

Figure S2. The MWIR 4X microscope lens used in the IR imaging. a Background thermal emission collected and blocked by the IR camera. b Close image of the heating stage and the lens. c Front view of the MWIR 4X microscope lens.

In this work, the IR images are taken by cooled IR cameras (with FPA sensors at $\left.T_{\text {cam }}=-193{ }^{\circ} \mathrm{C}\right)$ at the ambient temperature $T_{\text {env }}\left(=20^{\circ} \mathrm{C}\right)$ while the sample is heated 
to $T_{\text {dev }}\left(=80^{\circ} \mathrm{C}\right)$. According the Planck's law, the radiant exitance of blackbodies at $T_{\text {cam }}, T_{\text {env }}$ and $T_{\text {dev }}$ are calculated and integrated in the spectral response ranges of MWIR and LWIR bands:

\begin{tabular}{|c|c|c|}
\hline Radiant exitance & $\begin{array}{c}\text { Telops M200 } \\
(3.0 \mu \mathrm{m}-5.0 \mu \mathrm{m})\end{array}$ & $\begin{array}{c}\text { Telops L200 } \\
(7.7 \mu \mathrm{m}-9.3 \mu \mathrm{m})\end{array}$ \\
\hline$T=-193^{\circ} \mathrm{C}$ & $9.3 \times 10^{-12} \mathrm{~W} / \mathrm{m}^{2}$ & $2.4 \times 10^{-5} \mathrm{~W} / \mathrm{m}^{2}$ \\
\hline$T=20^{\circ} \mathrm{C}$ & $9.1 \mathrm{~W} / \mathrm{m}^{2}$ & $82.4 \mathrm{~W} / \mathrm{m}^{2}$ \\
\hline$T=80^{\circ} \mathrm{C}$ & $61.4 \mathrm{~W} / \mathrm{m}^{2}$ & $221.2 \mathrm{~W} / \mathrm{m}^{2}$ \\
\hline
\end{tabular}

In the experiments, all IR images are taken directly above the sample (Figure S2a). Since the radiative metasurface devices are smooth on the surface, background thermal emission is specularly reflected. Thus, the thermal emission from the environment $L_{\text {env }}\left(\lambda, \theta, T_{\text {env }}\right)$ can be neglected since it can not be collected by the lens. Since the thermal emission is largely enhanced when the radiative metasurface devices are heated to $80{ }^{\circ} \mathrm{C}$, the IR light emitted by the radiative metasurface devices are dominant among the IR signal captured by IR cameras. 
S2.2 Definition and estimation of the fill factor $f$ :
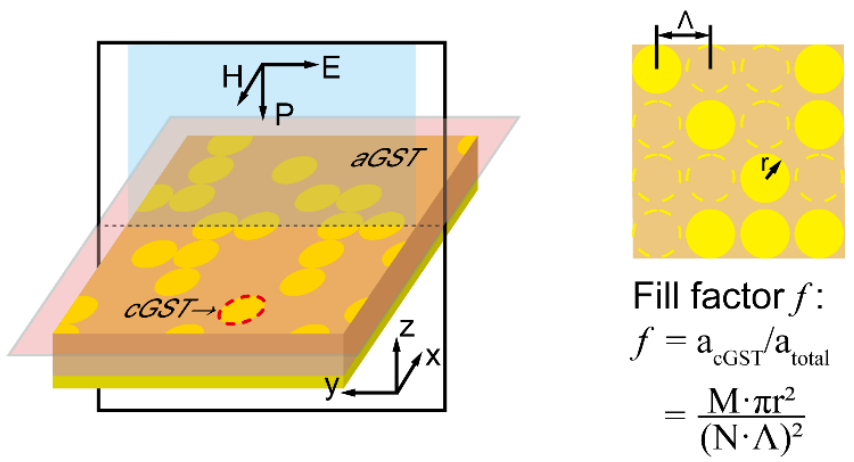

Figure S3. Sketch of the metasurface.

The fill factor $f$ is numerically defined to express the volume fraction of cGST among the GST film. For simplicity, the cGST spots are modeled as circle disks without overlap (Figure S3) so that the fill factor is given as $f=\frac{M \cdot \pi r^{2}}{(N \cdot \Lambda)^{2}}$. With the assumptions above, the $f$ is proportion to the number of cGST spots and has an upper $\operatorname{limit}$ of $\pi / 4(\sim 0.78)$.

The estimation of $f$ is based on the SEM images (Figure 2e and Figure S4a) where the amorphous GST (aGST) material and the crystalline GST (cGST) material exhibit different gray scales. To separate aGST and cGST spots, thresholding process is performed based on histograms (Figure S4b). Pixels representing aGST and cGST are assumed to be normally distributed in gray scale values in SEM images. Thus, the histogram can be fitted by two types of normal distribution curves which correspond to pixels of aGST and cGST areas. The threshold of segmentation is defined as

$$
\mu_{\text {threshold }}=\frac{\mu_{\mathrm{A}} \sigma_{\mathrm{B}}+\mu_{\mathrm{B}} \sigma_{\mathrm{A}}}{\sigma_{\mathrm{A}}+\sigma_{\mathrm{B}}}
$$


where $\mu_{\mathrm{A}}, \mu_{\mathrm{B}}, \sigma_{\mathrm{A}}$ and $\sigma_{\mathrm{B}}$ are the expectations and standard deviations of the normal distributions of type A and type B pixels, respectively. With these threshold values, segmentation results are shown in Figure S4c where white regions and black regions correspond to crystallized GST and as-deposited amorphous GST, respectively.

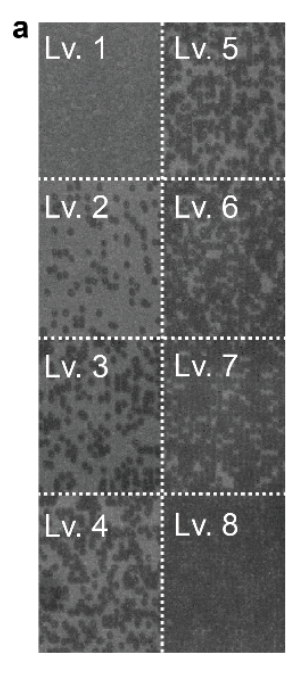

SEM images
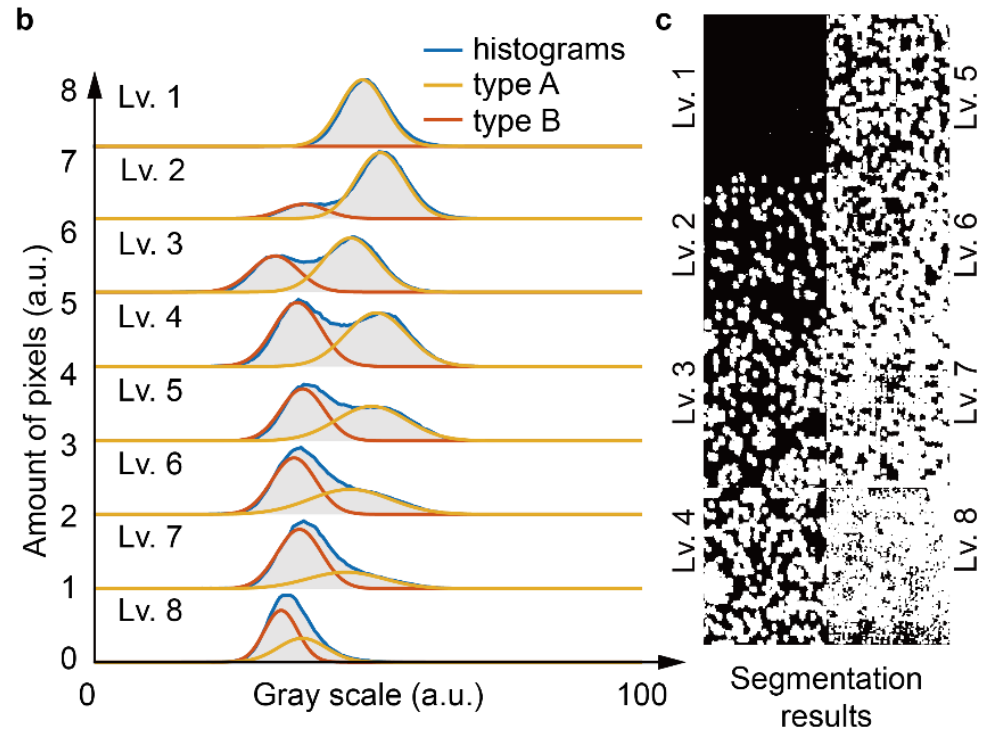

Figure S4 Thresholding process based on SEM images. (a) SEM images of regions written in Level 1 to 8. (b) Histograms of SEM images and two types of pixels fitted by the normal distribution. (c) Segmentation results from the thresholding process. 
S2.3 Multi-level control of optical response:

In this work, the multi-level control of IR spectral absorptance/emissivity is realized by controlling the fill factor of cGST among the GST layer. In practice, the fill factor $\mathrm{f}$ is controlled by the numbers of cGST spots in a square area $(80 \mu \mathrm{m} \times 80 \mu \mathrm{m})$.

Using the Maxwell-Garnett approximation ${ }^{\mathrm{S} 4}$, the GST layer which consists of cGST spots and non-crystallized aGST is substituted by a uniform layer with a permittivity $\varepsilon_{\text {eff }}$ where

$$
\varepsilon_{e f f}=\varepsilon_{\mathrm{aGST}} \frac{\varepsilon_{\mathrm{aGST}}+\frac{1+2 f}{3}\left(\varepsilon_{\mathrm{cGST}}-\varepsilon_{\mathrm{aGST}}\right)}{\varepsilon_{\mathrm{aGST}}+\frac{1-f}{3}\left(\varepsilon_{\mathrm{cGST}}-\varepsilon_{\mathrm{aGST}}\right)}
$$

With this approximation, the absorptance of the metasurface is calculated using the transfer matrix method ${ }^{\mathrm{S5}}$. Visible reflectance (Figure S6a) and chromaticity coordinates (Figure S6c) can also be calculated via this approach. Color differences are neglectable among these eight levels.
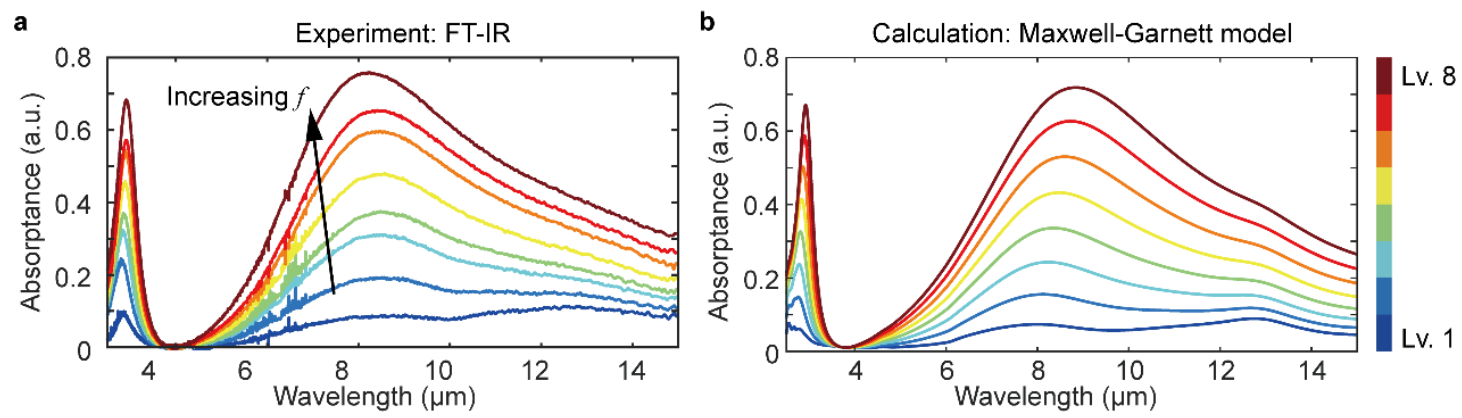

Figure S5. IR absorptance changes along the increase of fill factor f. (a). Experimental (b). Calculated results using Maxwell-Garnett model and transfer matrix method. 

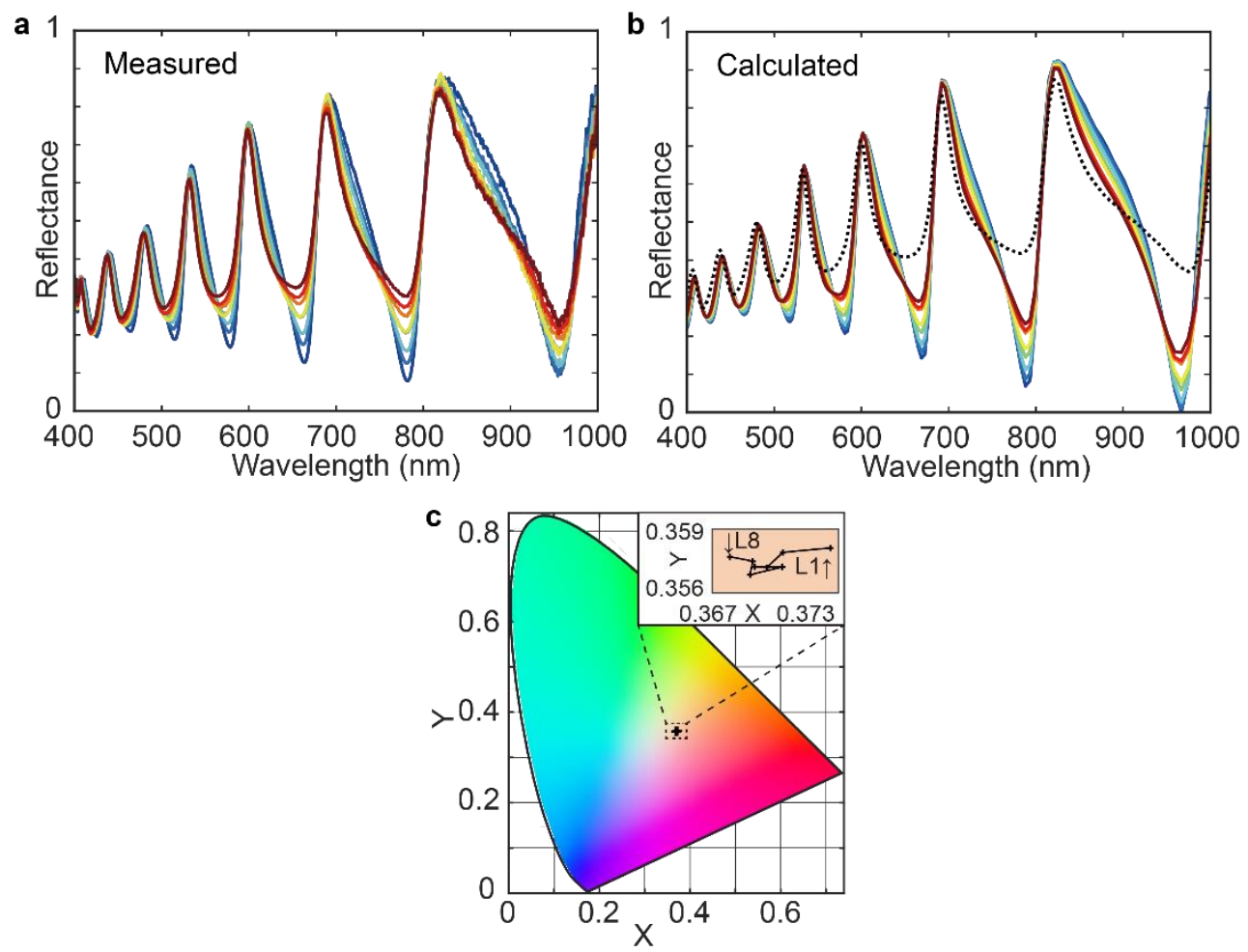

Figure S6 (a) Measured reflectance, (b) calculated reflectance and (c) calculated chromaticity coordinates in CIE1931 color space of Level 1 to 8. 
S2.4 Electric field distribution and resistive loss distribution:

a). Calculation of the IR absorption

To analysis the absorption of the radiative metasurfaces, especially how the fill factor $f$ affects the total absorption of the device, full-wave simulations of devices with fill factors varying from 0 to 0.78 (Lv. 1 to 8 ) are performed using the commercial FDTD software (Lumerical FDTD). In the FDTD simulations, the cGST spots are randomly placed without overlap. The whole simulation region is a $12 \mu \mathrm{m} \times$ $12 \mu \mathrm{m} \times 17 \mu \mathrm{m}$ box while field monitors only collect the electric and magnetic field components at central parts of cross sections. The incident plane wave is injected along the $-z$ direction while the electric field is $y$ polarized (Figure S3). Periodic boundary conditions are applied in $x$ and $y$ direction to reduce the computation complexity while the boundary conditions along $z$ direction are set as perfect matched layers. With the calculated normalized electric field $|\mathrm{E}|$ distributions (Figure S7), the resistive loss $Q$ distributions are further extracted (Figure $\mathrm{S} 8$ ). 


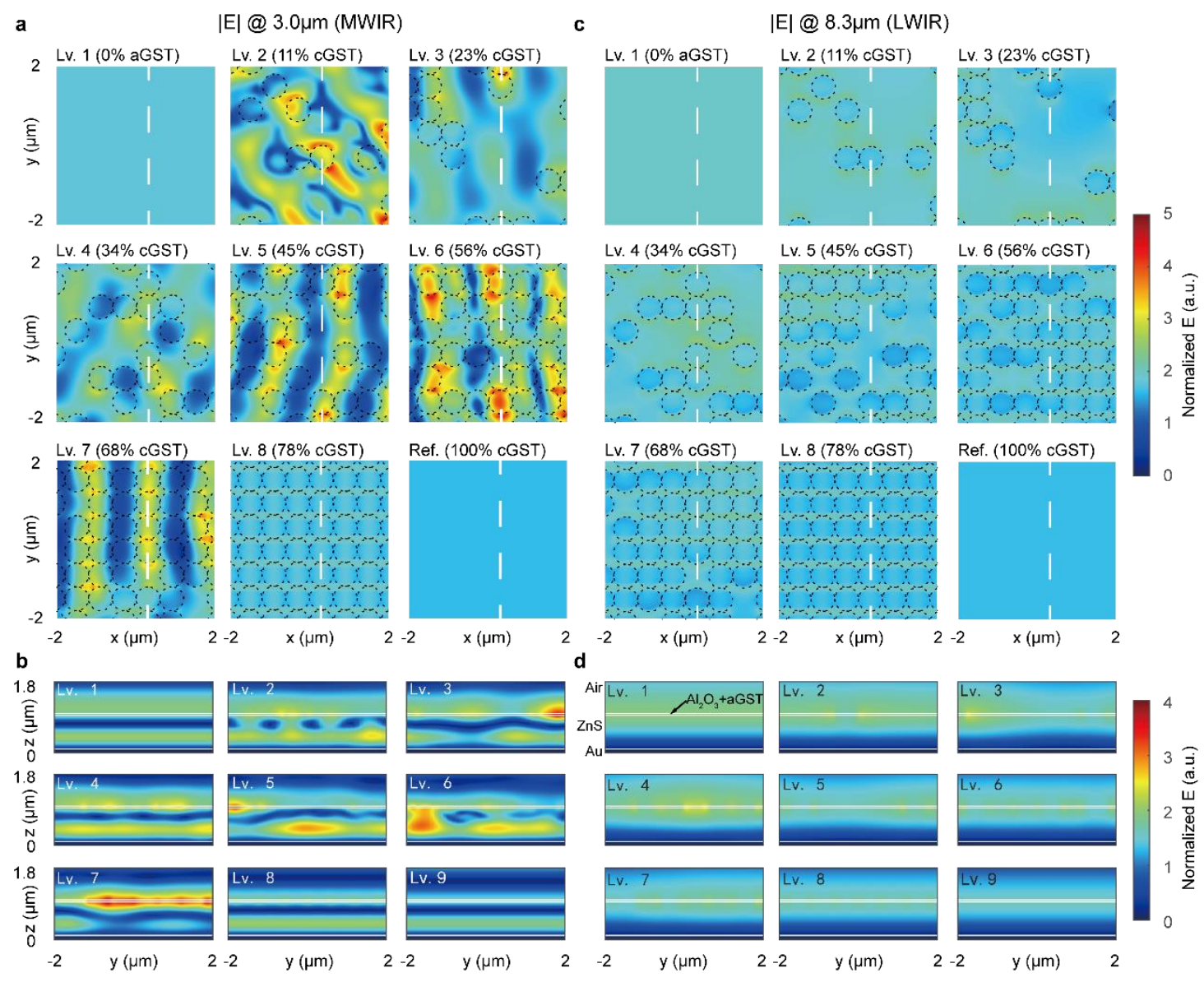

Figure S7. Normalized electric field $|\mathrm{E}|$ distributions of metasurfaces with different fill factors. a-b The normalized electric field $|\mathrm{E}|$ distributions on the surface and the cross section region of GST layer at the wavelength of $3.0 \mu \mathrm{m}$, respectively. c-d The normalized electric field $|\mathrm{E}|$ distribution on the surface and the cross section region of GST layer at the wavelength of $8.3 \mu \mathrm{m}$, respectively. 


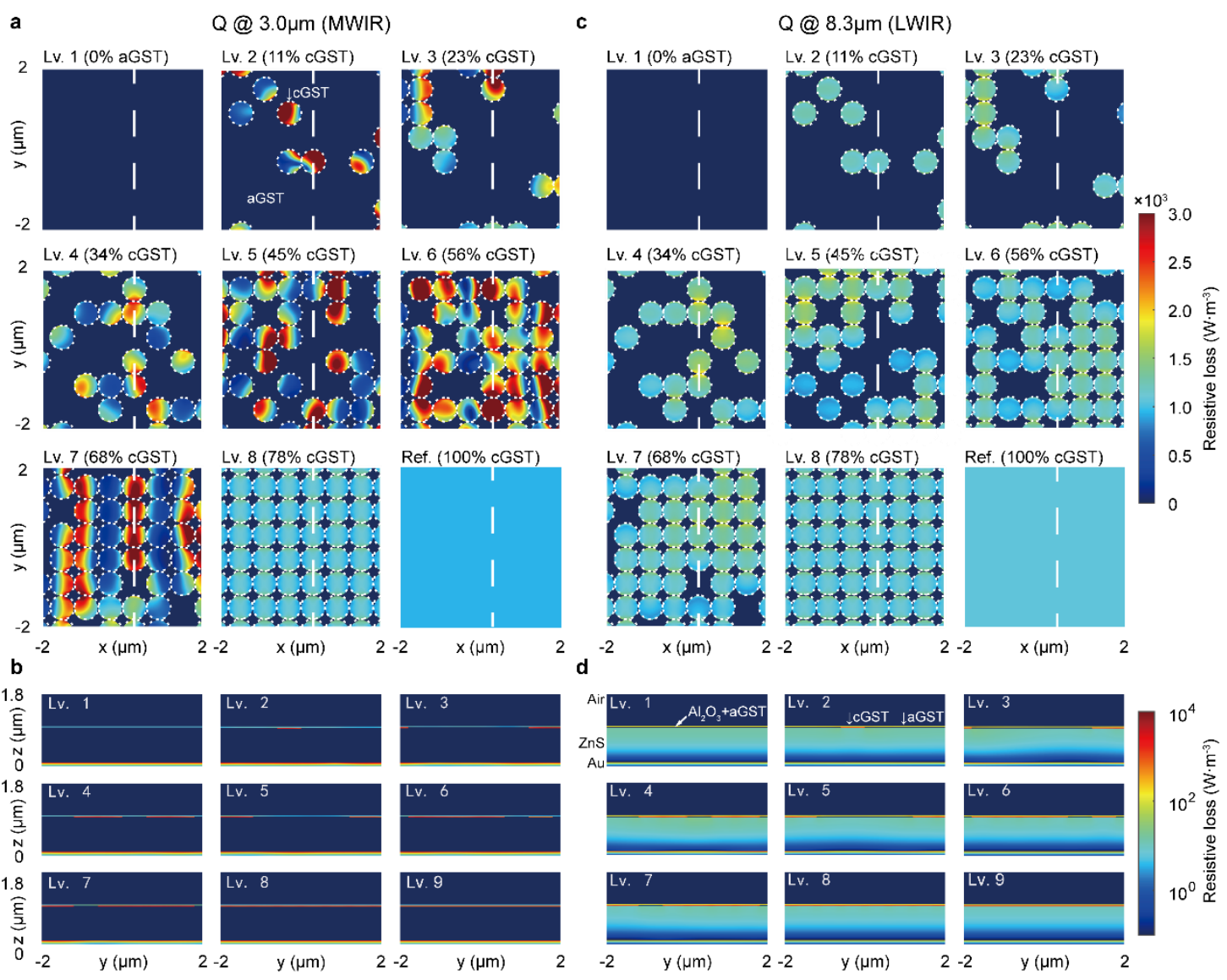

Figure S8. Resistive loss Q distributions of metasurfaces with different fill factors. a-

b The resistive loss Q distributions on the surface and the cross section region of GST layer at the wavelength of $8.3 \mu \mathrm{m}$, respectively. c-d The resistive loss Q distribution on the surface and the cross section region of GST layer at the wavelength of $8.3 \mu \mathrm{m}$, respectively.

b). Calculation of visible and IR scattering

To analysis the scattering of the sub-micron-sized bump, a single sub-micron-sized bump is modeled and calculated using the FDTD method. Firstly, situation where GST inside the bump is completely deposited on the $\mathrm{Al}_{2} \mathrm{O}_{3}$ (Figure S9a, Case I) is modeled and simulated. Structures with GST deposited inside the $\mathrm{Al}_{2} \mathrm{O}_{3}$ shell show apparent scattering in the calculated electrical field distribution regardless of the 
phase of GST (Figure S9c). The model in Case I is further modified (Case II in Figure S9b and 6d) by assuming that the part of the GST is deposited inside the bump (can be seen in Figure S9b). The Total-Field Scattered-Field (TFSF) source, which separates the computation region into total field region and scattered field region, is applied to evaluate the scattering of the bump in the visible spectral band and the MIR spectral band. Both cases show similar scattering properties in terms of the resonance wavelength, indicating similar color. Therefore, models assumed in the simulation can represent the actual structure in analyzing the optical response of the device.
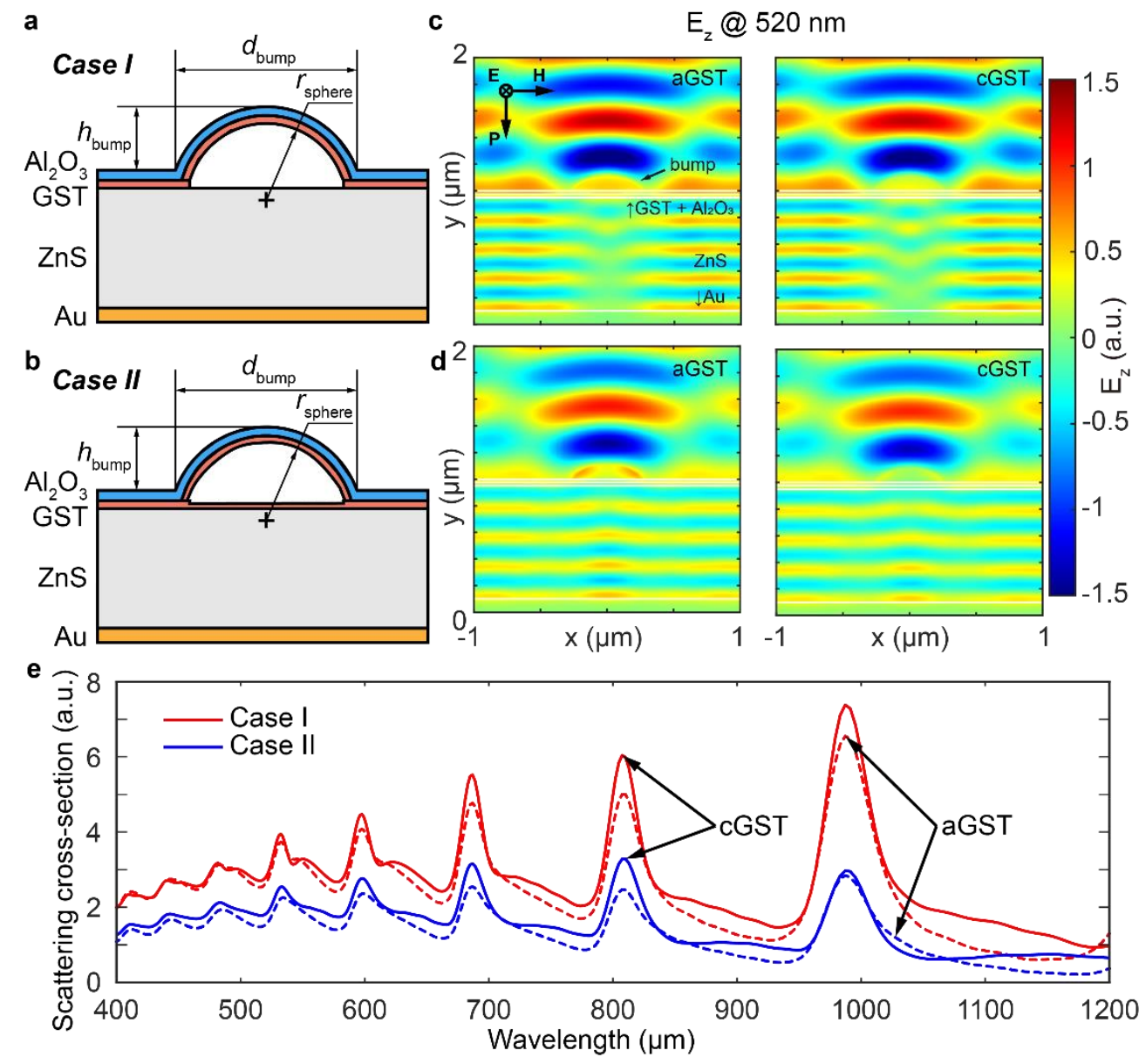

Figure S9 Simulation models and results. (a) Case I: GST completely deposited on A12O3. (b) Case II: GST uniformly deposited inside the bump. (c) - (d) Electrical 
field distribution of Case I, and II. (e) Scattering cross-section of the bumps in two cases. 
S2.5 Spectral responses of reversible phase transition:

a

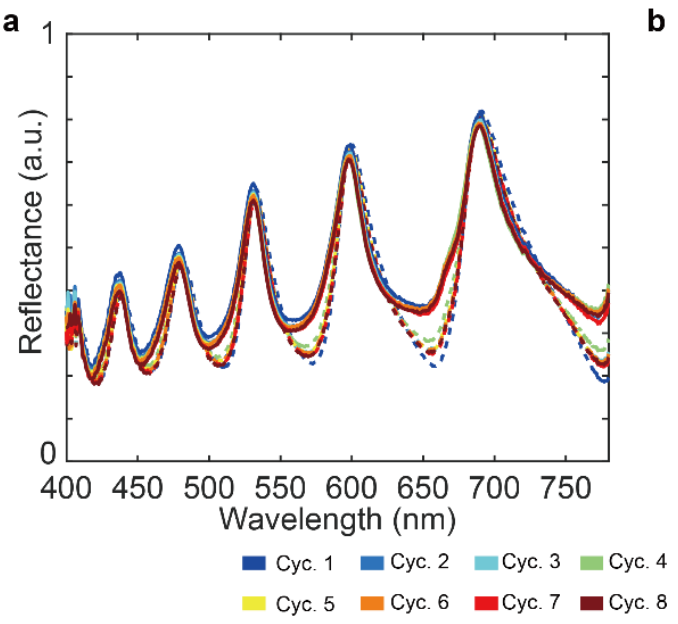

b

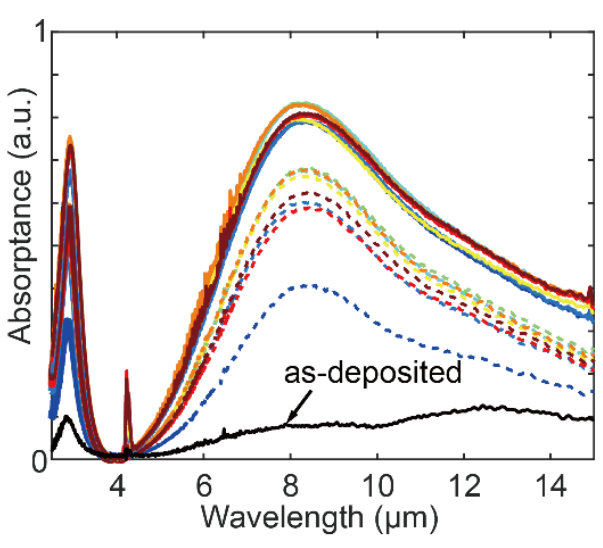

- After ms pulses (crystallization)

Figure S10 Optical characterization of the radiative metasurfaces after reversible phase transition of GST. (a) Visible reflectance and (b) IR absorptance after 1 to 8 cycles of crystallization and amorphization. 


\section{S2.6 Fluctuations in emissivity:}

The fluctuation in emissivity, which exhibits as "patterns" in IR images (see Figure S11 and Figure S12a), occur for various reasons. Firstly, defects inside the thin film, fragments such as dirt in the open air may increase the thermal emission, which are confirmed by SEM images, optical microscope images, and darkfield scattering microscope images, respectively (Figure S12b, c, and d, white arrows). Secondly, thickness non-uniformity of the thin films, especially the thickness non-uniformity of the GST layer, may also affect the total emissivity. As shown in Figure S14, peak absorptance increases $\sim 0.15$ while the thickness of GST increases from $20 \mathrm{~nm}$ to 30 nm. Thirdly, protective layer may undergo degradation after cycles of amorphization and crystallization, which may cause the oxidation or loss of GST and results in fluctuation of emissivity control. The distribution of non-uniformity corrected sensor readings for multilevel emissivity control (Figure S13a, corresponding to Figure 2b) and reversible emissivity control (Figure S13b, corresponding to Figure 2g) are demonstrated.
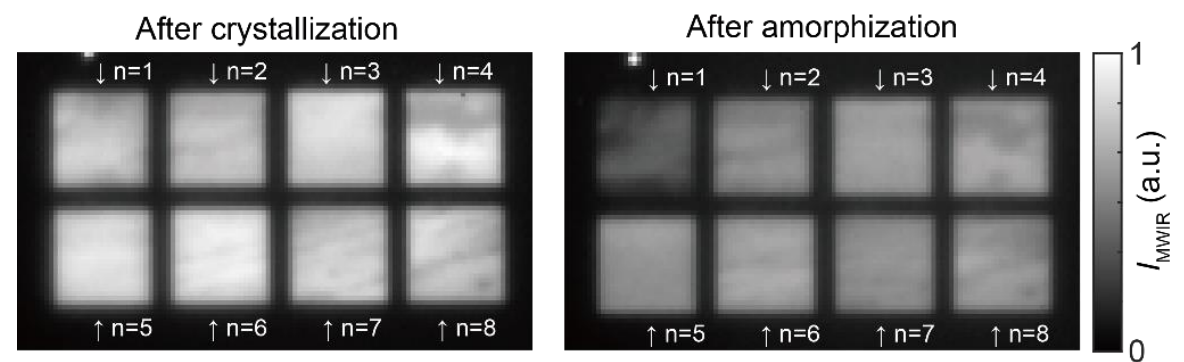

Figure S11 MWIR images of radiative metasurfaces after $n$ times $(n=1,2,3, \ldots, 8)$ of crystallization and amorphization (also shown in Figure 2g). 


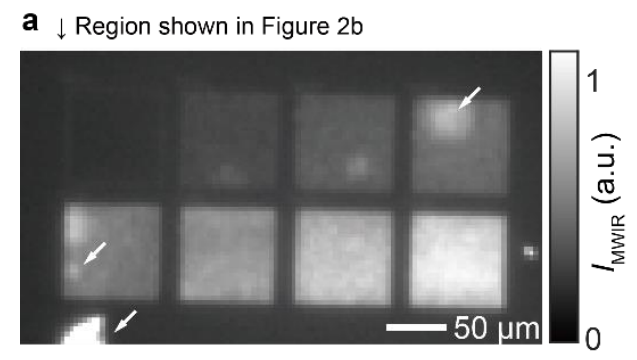

c

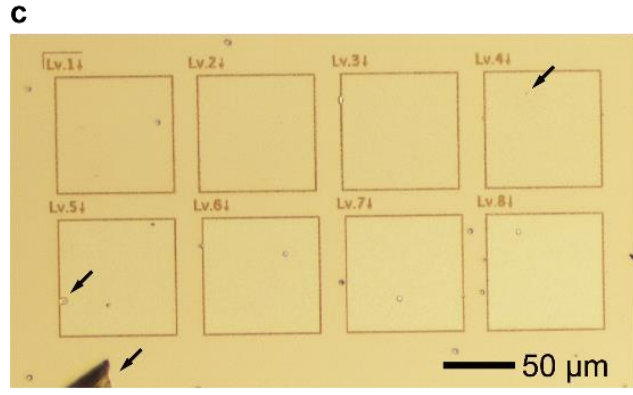

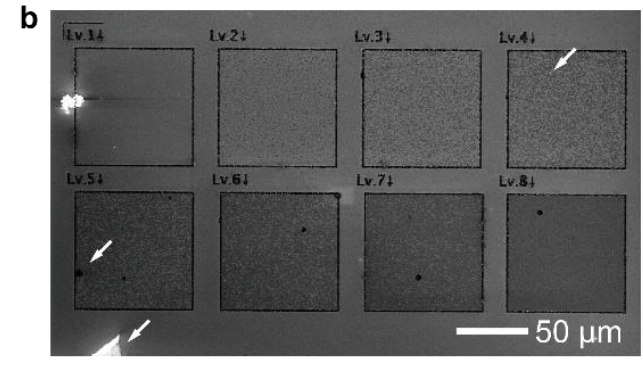

d

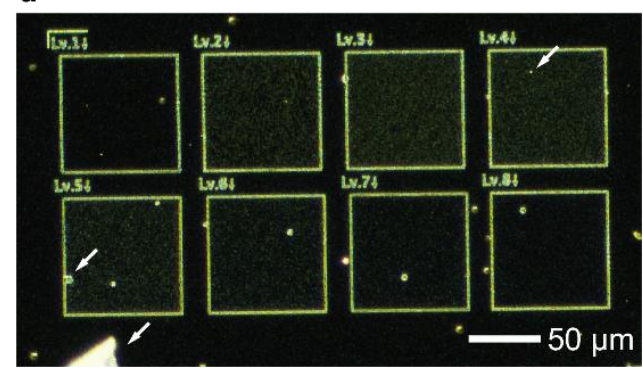

Figure S12 (a) MWIR images, (b) SEM images, (c) optical microscope images and (d) darkfield scattering images of multilevel radiative metasurfaces. White arrows in (a) indicate high emissivity regions. Arrows in (b-d) indicate defects inside the thin film and fragments.

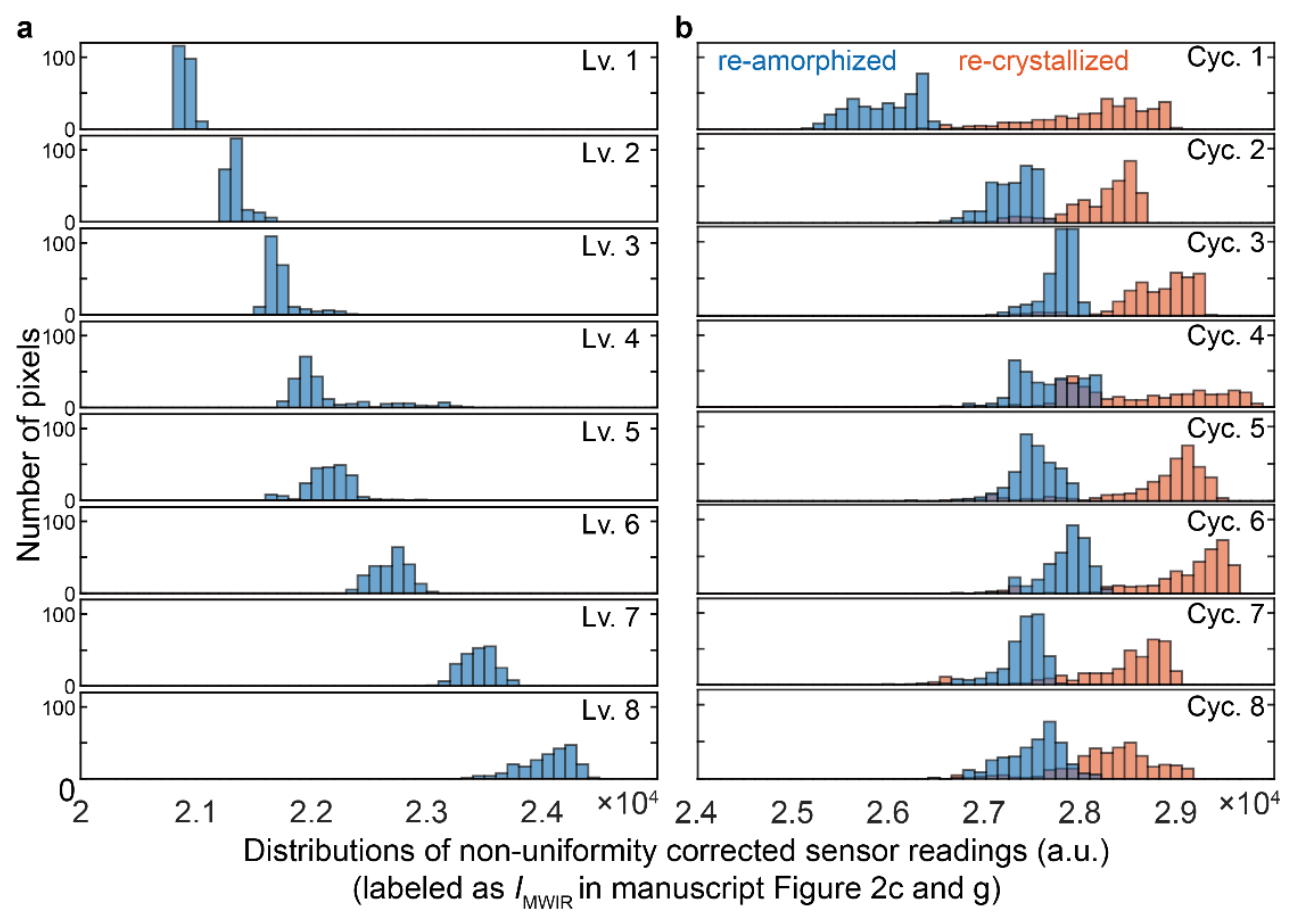

Figure S13 Distribution of non-uniformity corrected sensor readings corresponding to

(a) multilevel emissivity control in Figure $2 b$ and (b) reversible emissivity control in 
Figure S11 (or Figure 2g), respectively. The numbers of pixels for each metasurface are (a) $\sim 15 \times 15=225$ and (b) $\sim 19 \times 19=361$, respectively.

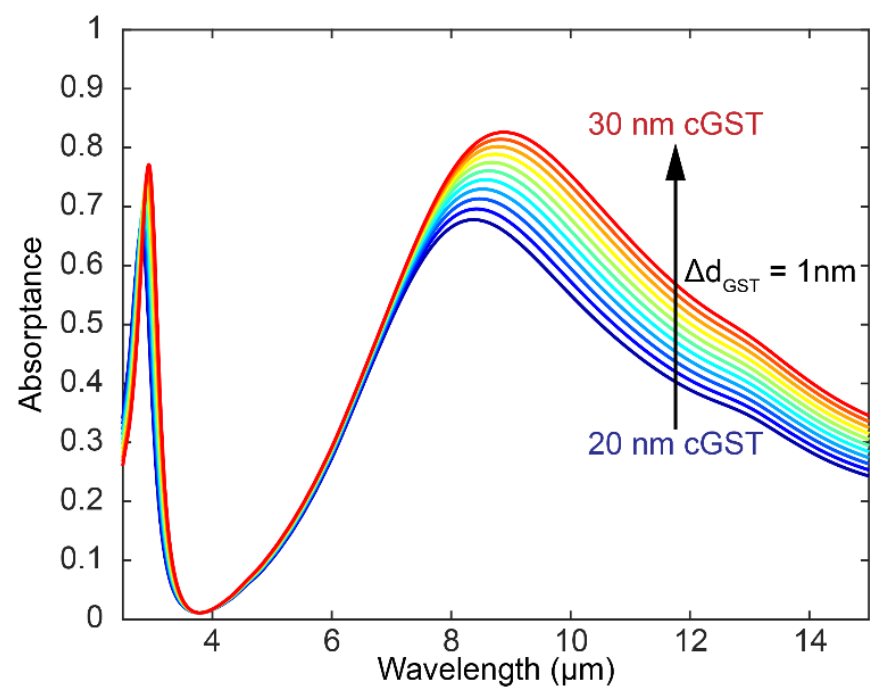

Figure S14 Absorptance at different thicknesses of the cGST layer 
S2.7 SEM images of areas encoded in Mode I, II, and III

a

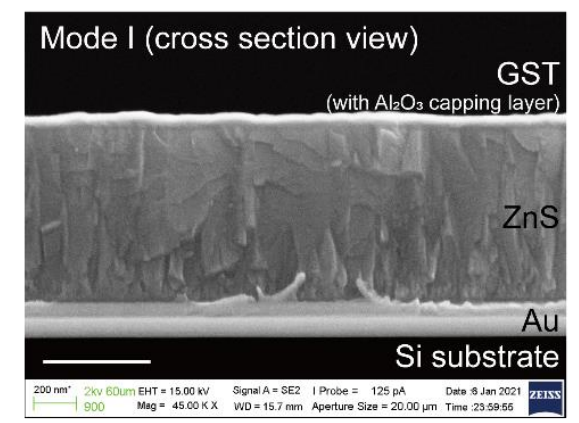

d

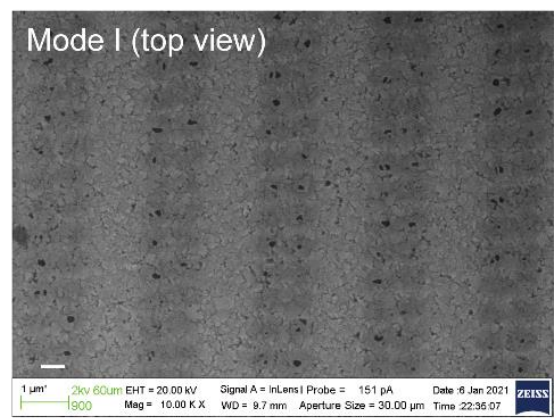

b
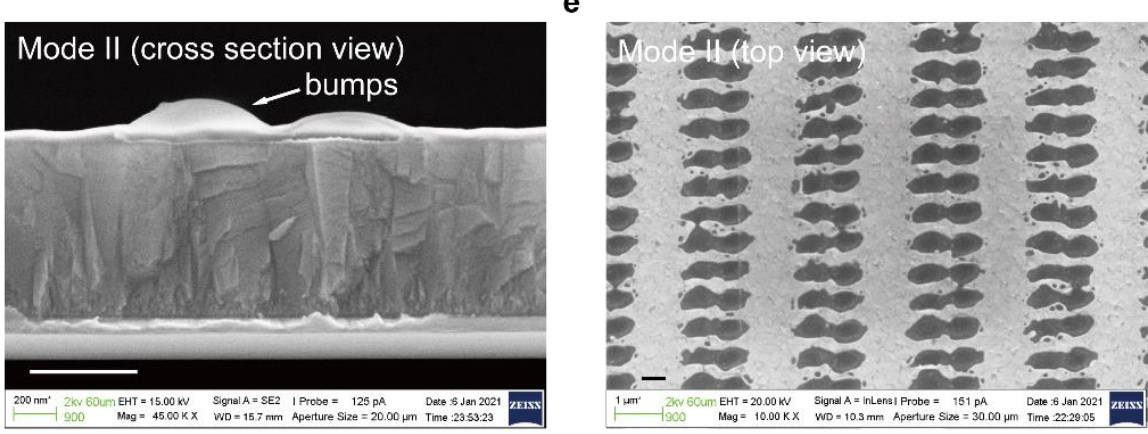

C
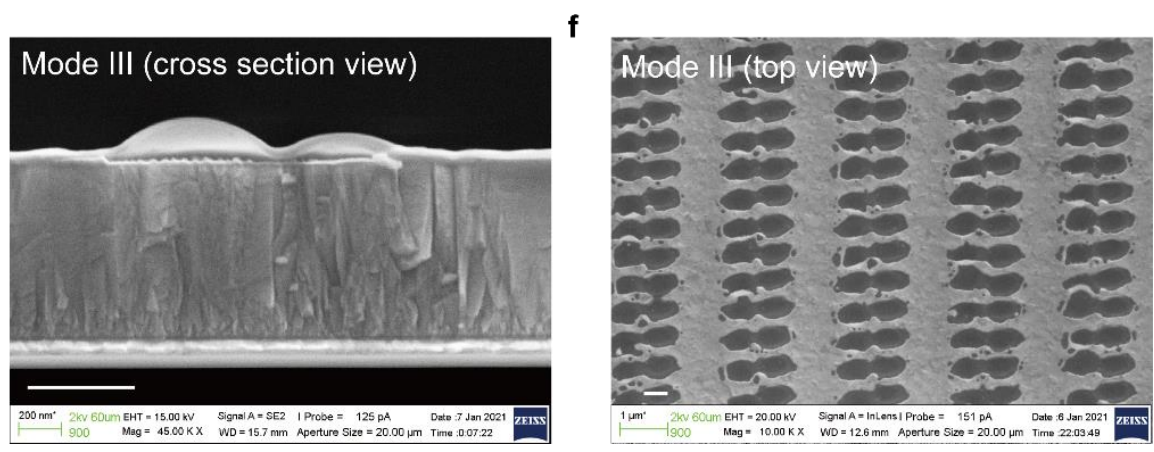

Figure S15. SEM images of the device patterned in Mode I, II, and III. a-c Cross section images of regions patterned in Mode I, II, and III, respectively. d-f Top view SEM images. Scale bars are $500 \mathrm{~nm}$.

Irradiated by the ms or ns laser pulses, the device exhibits changes in visible and IR patterns due to the phase transition of GST and the formation of sub-micron-sized bumps. For comparison, columns of dots are patterned with ms (Mode I), ns (Mode II), and $\mathrm{ms}+\mathrm{ns}$ (Mode III) laser pulses. The SEM images of the patterned region are shown in Figure S15, in the cross section view $(\mathrm{a}-\mathrm{c})$ and the top view (d-f). As 
shown in Figure S15, bumps only appear in Mode II and Mode III while no obvious change can be found in the cross section image of Mode I (Figure S15a). 
S2.8 Damage of surface without capping layer

The $\mathrm{Al}_{2} \mathrm{O}_{3}$ capping layer is essential in the formation of sub-micron-sized bumps.

For the device without the $\mathrm{Al}_{2} \mathrm{O}_{3}$ capping layer, obvious damages are noticed in the cross section SEM image (Figure S16a) and the top view SEM image (Figure S16b). As the comparison, SEM images of the device with the capping layer are presented in Figure S16c and Figure S16d.
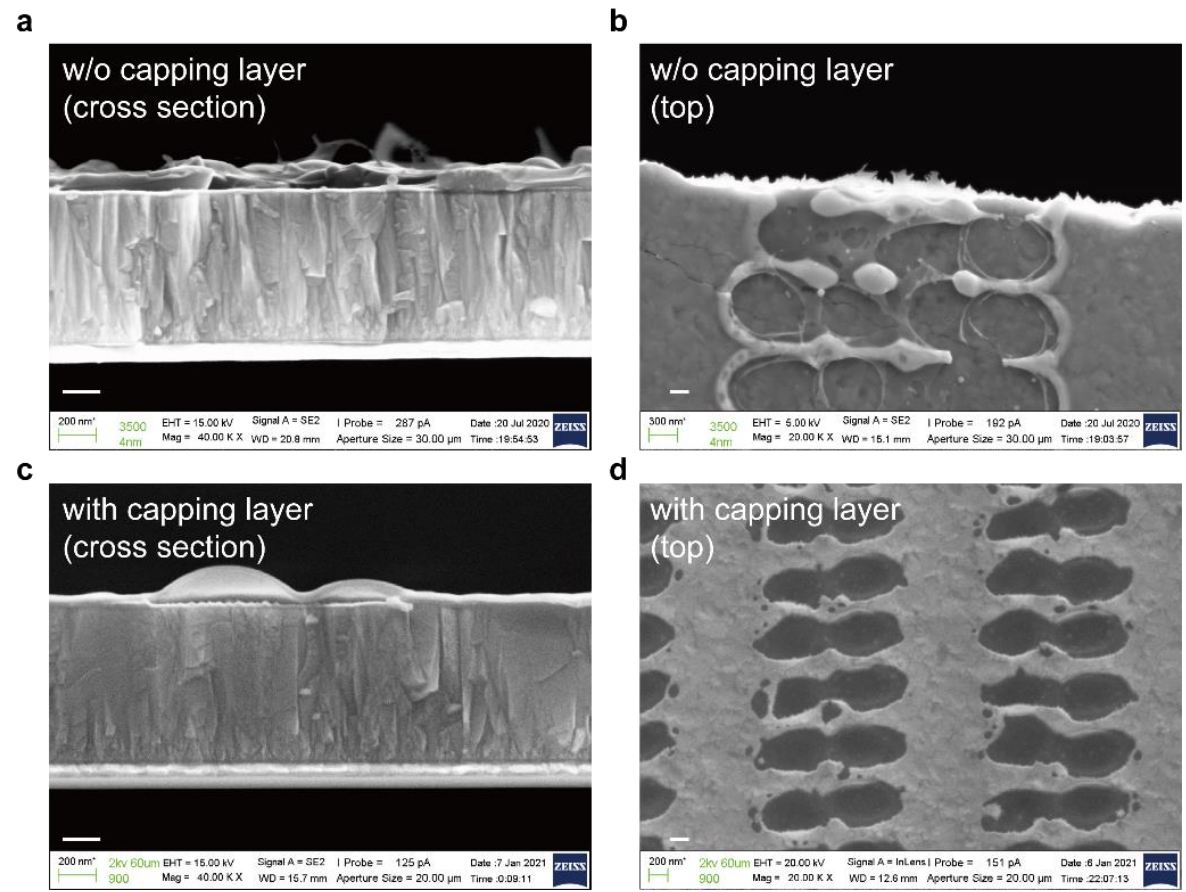

Figure S16. SEM images of devices with and without $\mathrm{Al}_{2} \mathrm{O}_{3}$ capping layer after highpower laser pulses irradiation. (a) Cross section and (b) top views of the device without capping layer. (c) Cross section and (d)top views of the device with capping layer. Scale bars are $200 \mathrm{~nm}$. 
S2.9 Generation of patterns for the anti-counterfeiting application

a

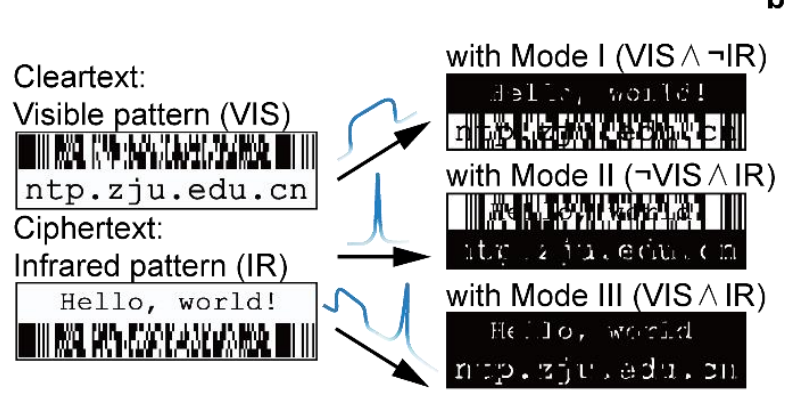

b

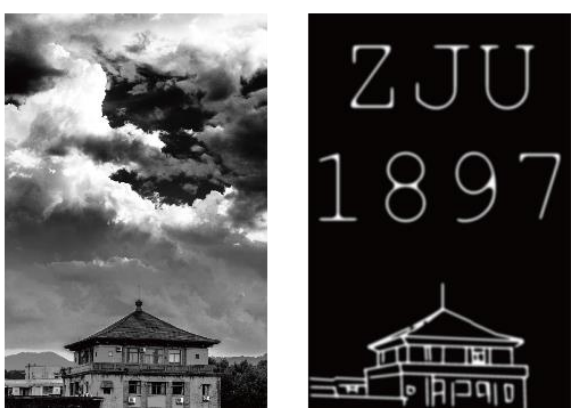

Figure S17. Generation of patterns for the anti-counterfeiting application. (a) Cleartext and cipher text and the converted to digital masks for laser direct writing processes with Mode I, II, and III. (b) The grayscale image of the No. 4 teaching building of Yuquan campus, Zhejiang University (left) is chosen as the cleartext while the text "ZJU 1897" (right) is chosen as the ciphertext.

In the generation of patterns for anti-counterfeiting applications, logical operations of the cleartext and the ciphertext are taken to define locations to be patterned with Mode I, II, and III (Figure S17a). Image of No. 4 teaching building in Yuquan campus, Zhejiang University (Figure $\mathrm{S} 17 \mathrm{~b}$ ) is also used in the demonstration of anticounterfeiting applications. 
Table S1. Table shows the comparison of spatially resolved control of thermal emission.

\begin{tabular}{|c|c|c|c|c|c|c|c|c|c|}
\hline Materials & Mechanism & $\begin{array}{l}\text { Volatile o } \\
\text { nonvolatile? }\end{array}$ & $\mid \begin{array}{l}\text { Static or } \\
\text { Reconfig } \\
\text { urable? }\end{array}$ & Stimulus & $\begin{array}{l}\text { Emissivity } \\
\text { modulation }\end{array}$ & $\begin{array}{l}\text { Control of visible } \\
\text { patterns }\end{array}$ & $\begin{array}{l}\text { Pixel } \\
\text { size }\end{array}$ & $\begin{array}{l}\text { Thickness } \\
\text { (w/o } \\
\text { substrate) }\end{array}$ & Ref. \\
\hline $\mathrm{Au} / \mathrm{SiO}_{2} / \mathrm{Au}$ & $\begin{array}{l}\text { Metal- } \\
\text { insulator-metal } \\
\text { resonators }\end{array}$ & \multirow{3}{*}{ Nonvolatile } & \multirow{3}{*}{ Static } & & & $\begin{array}{l}\text { Related to IR } \\
\text { patterns }\end{array}$ & $2 \mu \mathrm{m}$ & $-500 \mathrm{~nm}$ & S6 \\
\hline $\mathrm{Au} / \mathrm{SU} 8$ & $\begin{array}{l}\text { Imprinted } \\
\text { plasmonic } \\
\text { cavities }\end{array}$ & & & - & - & $\begin{array}{l}\text { Related to IR } \\
\text { patterns }\end{array}$ & $1.2 \mu \mathrm{m}$ & $\sim \mu \mathrm{m}$ & S7 \\
\hline $\mathrm{SiO}_{2} / \mathrm{CaF}_{2} / \mathrm{Al}$ & $\begin{array}{l}\text { Fabry-Perot } \\
\text { cavity with } \\
\mathrm{SiO}_{2}\end{array}$ & & & - & - & Independent & $25.4 \mu \mathrm{m}$ & $-2 \mu \mathrm{m}$ & S8 \\
\hline $\begin{array}{l}\mathrm{Au} / \mathrm{n} \text {-doped } \\
\mathrm{ZnO} / \mathrm{ITO}\end{array}$ & $\begin{array}{l}\text { Photocarrier } \\
\text { doping }\end{array}$ & Volatile & \multirow{3}{*}{$\begin{array}{l}\text { Reconfig } \\
\text { urable }\end{array}$} & Optical & $\begin{array}{l}0.60-0.76 \\
(\lambda=11.5 \mu \mathrm{m})\end{array}$ & Not reported & $0.6 \mu \mathrm{m}$ & $\sim 1 \mu \mathrm{m}$ & S9 \\
\hline $\mathrm{VO}_{2} / \mathrm{ZnS} / \mathrm{Au}$ & $\begin{array}{l}\text { Fabry-Perot } \\
\text { cavity with } \\
\mathrm{VO}_{2}\end{array}$ & Volatile & & $\begin{array}{l}\text { Optical/ } \\
\text { Thermal }\end{array}$ & $\begin{array}{l}0.19-0.91 \\
(\lambda \sim 10 \mu \mathrm{m})\end{array}$ & $\begin{array}{l}\text { Related to IR } \\
\text { patterns }\end{array}$ & $0.5 \mathrm{~mm}$ & $\sim 1 \mu \mathrm{m}$ & $\mathrm{S} 10$ \\
\hline $\begin{array}{l}\text { Thermochromic } \\
\text { liquid crystal }\end{array}$ & $\begin{array}{l}\text { Thermoelectric } \\
\text { units }\end{array}$ & Volatile & & Thermal & Not reported & $\begin{array}{l}\text { Related to IR } \\
\text { patterns }\end{array}$ & $6 \mathrm{~mm}$ & $\begin{array}{l}\text { a few hundred } \\
\text { micrometers }\end{array}$ & S11 \\
\hline
\end{tabular}




\begin{tabular}{|l|l|l|l|l|l|l|l|}
\hline $\begin{array}{l}\text { Multilayer } \\
\text { graphene/PE } \\
\text { ionic liquid/Au }\end{array}$ & $\begin{array}{l}\text { Gate-controlled } \\
\text { ion } \\
\text { intercalation } \\
\text { and } \\
\text { deintercalation }\end{array}$ & Nonvolatile & Electrical & $\begin{array}{l}0.33-0.76 \\
(\lambda=10 \mu \mathrm{m})\end{array}$ & Not reported & $2 \mathrm{~cm}$ & $<25 \mu \mathrm{m}$ \\
\hline GST/ZnS/Au & $\begin{array}{l}\text { Fabry-Perot } \\
\text { cavity with/Nonvolatile } \\
\text { GST }\end{array}$ & $\begin{array}{l}\text { Reconfig } \\
\text { urable }\end{array}$ & Optical & $\begin{array}{l}0.1-0.7 \\
(\lambda=8 \mu \mathrm{m})\end{array}$ & Independent & $0.6 \mu \mathrm{m}$ & $\sim 1 \mu \mathrm{m}$ \\
\hline
\end{tabular}

Note:

${ }^{\square}$ IR patterns appear as uniform areas of color in the visible domain so that the information is hidden in IR domain.

${ }^{\diamond}$ Distinguishable patterns appear in visible domain, which are same as the IR patterns. 


\section{References}

S1. Smith, D.; Shiles, E.; Inokuti, M.; Palik, E., Handbook of optical constants of solids. Academic Press: San Diego, CA, 1985; Vol. 1.

S2. Querry, M., Optical constants of minerals and other materials from the millimeter to the ultraviolet. Chemical Research Development and Engineering Center: Aberdeen Proving Ground, MD, 1987.

S3. Du, K.; Li, Q.; Lyu, Y.; Ding, J.; Lu, Y.; Cheng, Z.; Qiu, M., Control over emissivity of zero-static-power thermal emitters based on phase-changing material GST. Light Sci. Appl. 2017, $6(1), \mathrm{e} 16194$.

S4. Markel, V. A., Introduction to the Maxwell Garnett approximation: tutorial. J. Opt. Soc. Am. A 2016, 33 (7), 1244-56.

S5. Pettersson, L. A. A.; Roman, L. S.; Inganäs, O., Modeling photocurrent action spectra of photovoltaic devices based on organic thin films. J. Appl. Phys. 1999, 86 (1), 487-496.

S6. Makhsiyan, M.; Bouchon, P.; Jaeck, J.; Pelouard, J.-L.; Haïdar, R., Shaping the spatial and spectral emissivity at the diffraction limit. Appl. Phys. Lett. 2015, 107 (25), 251103.

S7. Franklin, D.; Modak, S.; Vazquez-Guardado, A.; Safaei, A.; Chanda, D., Covert infrared image encoding through imprinted plasmonic cavities. Light Sci. Appl. 2018, 7, 93.

S8. Bakan, G.; Ayas, S.; Serhatlioglu, M.; Elbuken, C.; Dana, A., Invisible Thin-Film Patterns with Strong Infrared Emission as an Optical Security Feature. Adv. Opt. Mat. 2018, 6 (21), 1800613. 
S9. Coppens, Z. J.; Valentine, J. G., Spatial and Temporal Modulation of Thermal Emission. Adv. Mat. 2017, 29 (39), 1701275.

S10. Xu, Z.; Li, Q.; Du, K.; Long, S.; Yang, Y.; Cao, X.; Luo, H.; Zhu, H.; Ghosh, P.; Shen, W.; Qiu, M., Spatially Resolved Dynamically Reconfigurable Multilevel Control of Thermal Emission. Laser Photonics Rev. 2019, 14 (1), 1900162.

S11. Lee, J.; Sul, H.; Jung, Y.; Kim, H.; Han, S.; Choi, J.; Shin, J.; Kim, D.; Jung, J.; Hong, S.; Ko, S. H., Thermally Controlled, Active Imperceptible Artificial Skin in Visible - to - Infrared Range. Adv. Funct. Mater. 2020, 30 (36), 2003328.

S12. Salihoglu, O.; Uzlu, H. B.; Yakar, O.; Aas, S.; Balci, O.; Kakenov, N.; Balci, S.; Olcum, S.; Suzer, S.; Kocabas, C., Graphene-Based Adaptive Thermal Camouflage. Nano Lett. 2018, 18 (7), 4541-4548. 